anales de psicología, 2013, vol. 29, n ${ }^{\circ} 3$ (octubre), 724-733 http://dx.doi.org/10.6018/analesps.29.3.151041
(C) Copyright 2013: Servicio de Publicaciones de la Universidad de Murcia. Murcia (España) ISSN edición impresa: 0212-9728. ISSN edición web (http://revistas.um.es/analesps): 1695-2294

\title{
Riesgo de trastorno de la conducta alimentaria, consumo de sustancias adictivas y dificultades emocionales en chicas adolescentes
}

\author{
María S. Cruz-Sáez ${ }^{1 *}$, Aitziber Pascual ${ }^{1}$, Itziar Etxebarria ${ }^{1}$ y Enrique Echeburúa ${ }^{12}$ \\ 1 Facultad de Psicología. Universidad del País V asco UPV-EHU \\ 2 Cibersam
}

Resumen: El objetivo del presente trabajo fue estimar la prevalencia de
riesgo de trastorno de la conducta alimentaria en una muestra de 767 muje-
res de 16 a 20 años, así como estudiar las diferencias entre las adolescentes
de alto y bajo riesgo en diferentes variables (sociodemográficas, antro-
pométricas, percepción de la imagen corporal, sobrevaloración de la delga-
dez, consumo de sustancias, alexitimia y ansiedad). La prevalencia de muje-
res adolescentes de alto riesgo fue del $15 \%$. Según los resultados obteni-
dos, el peso, el índice de masa corporal, la percepción de la imagen corpo-
ral y la sobrevaloración de la delgadez eran factores relevantes en las ado-
lescentes con alto riesgo de trastorno alimentario. Asimismo un mayor
porcentaje de adolescentes de alto riesgo fumaban, habían probado drogas
de síntesis y habían empezado antes a consumir alcohol. También se obtu-
vieron mayores niveles de alexitimia y ansiedad en estas adolescentes de al-
to riesgo. Estos resultados sugieren que la prevención de los trastornos
alimentarios debería incluir, además de educación nutricional e informa-
ción sobre el efecto nocivo de la interiorización del ideal de delgadez ex-
trema imperante, técnicas de expresión y regulación emocional a fín de que
no tengan que recurrir a conductas inadecuadas de control del peso y de la
imagen corporal.
Palabras clave: Riesgo de trastorno alimentario; consumo de sustancias; Palabras clave: Riesgo de trasto
alexitimia; ansiedad; adolescencia.
Title: Risk of eating disorders, consumption of addictive substances and emotional difficulties in adolescent girls.

Abstract: The aim of this study was to estimate the prevalence of risk of developing eating disorders in a sample of 767 adolescent girls aged between 16 and 20, and to study the differences between adolescent girls at high risk and low risk of developing eating disorders in relation to different variables (sociodemographic, anthropometric, body image perception, overvaluation of thinness, substance consumption, alexithymia and anxiety). The prevalence of adolescent girls at high risk was found to be $15 \%$. The results revealed that weight, BMI, body image perception and overvaluation of thinness were key factors in adolescent girls at high risk of developing eating disorders. Also, a higher percentage of at high risk adolescent girls smoked, had tried synthetic drugs and started drinking alcohol at a younger age. Higher levels of alexithymia and anxiety were also found in at-risk adolescents. These results suggest that, in addition to nutritional education and information about the effects of internalizing the thin ideal on adolescent personalities, eating disorders prevention programs should also include techniques for expressing and regulating emotions, in order to prevent young girls from turning to inappropriate weight and body image control behaviors.

Key words: risk of eating disorders, substance consumption, alexithymia, anxiety, adolescence.

\section{Introducción}

Los trastornos de la conducta alimentaria (TCA), que, según el DSM-IV-TR (American Psychiatric Association, 2000), incluyen la anorexia nerviosa, la bulimia y los TCA no especificados, se definen como una mala regulación del comportamiento alimentario, caracterizada por alteraciones cognitivas y conductuales asociadas a una preocupación excesiva por el peso y la imagen corporal (Overton, Selway, Strongman y Houston, 2005; Schmidt y Treasure, 2006). Las pacientes con TCA se caracterizan por una búsqueda deliberada de pérdida de peso a través de comportamientos de restricción/ purga alimentaria y/o excesivo ejercicio.

La cultura actual promueve la delgadez en la mujer como sinónimo de belleza, aceptación y éxito, de tal forma que una buena parte de las chicas adolescentes muestran insatisfacción con su forma y peso corporal. Esta situación puede desencadenar una serie de comportamientos inadecuados relacionados con la alimentación y el peso, que suponen un riesgo para el desarrollo de un trastorno alimentario (Stice y Shaw, 2010; Stice, Marti y Durant, 2011). De hecho, en los últimos años la prevalencia de los TCA y de los problemas relacionados con el peso (sobrepeso, atracones con o sin vómitos, actitudes y comportamientos dirigidos al control del

* Dirección para correspondencia [Correspondence address]:

María Soledad Cruz-Sáez. Universidad del País Vasco. Facultad de Psicología. Avenida de Tolosa, 70. 20018 San Sebastián, Guipúzcoa (España). E-mail: mariasoledad.cruz@,ehu.es peso, etcétera) han aumentado considerablemente en la población adolescente (Neumark-Sztainer et al., 2007; Sancho, Arija, Asorey y Canals, 2007). Según la bibliografía existente, la prevalencia para el riesgo de TCA en chicas estudiantes se sitúa entre el 7.3 y el 18\% (revisado por Sepúlveda, Carrobles y Gandarillas, 2008). Aunque tales actitudes y comportamientos no constituyan propiamente un cuadro clínico, pueden, sin embargo, poner en riesgo la salud, sobre todo en esta etapa evolutiva (González, Penelo, Gutiérrez y Raich, 2010).

Sentirse insatisfecha con la imagen corporal supone un factor de riesgo importante en los TCA, ya que las actitudes hacia el propio cuerpo afectan tanto a la conducta como a los pensamientos y a las emociones (Gasco, Briñol y Horcajo, 2010). En concreto, el índice de masa corporal (IMC) es uno de los factores que más afectan a la insatisfacción corporal y a las alteraciones alimentarias (Espinoza, Penelo y Raich, 2010). Así, los valores elevados de IMC se asocian a un aumento de la preocupación por la imagen y forma corporal, a la insatisfacción con el cuerpo, a la sintomatología depresiva y a conductas poco saludables de control del peso (Crow, Eisenberg, Story y Neumark-Sztainer, 2006; Wardle y Cooke, 2005). Otros factores que pueden desempeñar un papel importante en el desarrollo de los TCA son los estresores sociales, tales como los derivados del nivel socioeconómico, el grado de escolaridad y la ocupación laboral de los padres (Hunot, Vizmanos, Vázquez-Garibay y Celis, 2008). 
Los TCA resultan de gran interés, no solo por las graves alteraciones físicas y psicológicas que conllevan, sino también por la posible relación que guardan con otros cuadros clínicos, entre los que se encuentra el consumo de drogas. Los TCA se presentan principalmente en mujeres adolescentes, siendo, a su vez, la adolescencia la edad de mayor riesgo para iniciarse en la ingesta de sustancias adictivas. El que las adolescentes adopten ciertas conductas alimentarias anómalas para controlar su peso puede ser un incentivo para utilizar algún tipo de drogas (Krahn, Kurth, Demitrack y Drewnoski, 1992). Hay abundante bibliografía que muestra una relación positiva entre el consumo de sustancias adictivas y los TCA (Calero-Elvira et al., 2009). Así, en algunos estudios se ha visto que fumar tabaco puede utilizarse como forma de reducir el apetito y, por tanto, como estrategia de reducción del peso (Anzengruber et al., 2006; Quiles-Marcos et al., 2011). Los trabajos que han evaluado la relación entre el consumo de alcohol en adolescentes y el riesgo de desarrollar un TCA han hallado diferencias entre adolescentes de alto y bajo riesgo (Gadalla y Piran, 2007; Luce, Engler y Crowther, 2007; Quiles-Marcos et al., 2011). En cuanto a otras drogas, si bien hay estudios que indican que su consumo está relacionado con un mayor riesgo de desarrollar un TCA (Gutiérrez, Mora, Unikel, Villatoro y Medina-Mora, 2001; Herrero, Viña y Ramírez, 2010; Gadalla y Pirán, 2007), también hay estudios recientes que no hallan dicha relación (Bisetto, Botella y Sancho, 2012; Quiles-Marcos et al., 2011).

Asimismo los TCA se asocian a altos niveles de comorbilidad con trastornos de ansiedad, conductas impulsivas y trastornos de personalidad (Marañón, Echeburúa y Grijalvo, 2004, 2007). En este sentido, como hemos señalado, se podría considerar a los TCA como estrategias de regulación emocional inadecuadas. Así, las personas con un TCA pueden tener dificultades importantes en el manejo y regulación de las emociones, especialmente de las negativas (Hayaki, 2009; Pascual, Etxebarria, Cruz y Echeburúa, 2011; Waller et al., 2010). Entre las variables emocionales que más interés han despertado en el estudio de los TCA se encuentran la alexitimia y la ansiedad. Ya Bruch $(1962,1981)$ sugirió que la dificultad para discriminar y expresar sentimientos, así como para diferenciar estados emocionales y sensaciones corporales, constituía un déficit central en los trastornos alimentarios. Estudios posteriores han confirmado la relación entre la alexitimia y los TCA (Gilboa-Schechtman, Avnon, Zubery y Jeczmien, 2006; Hayaki, Friedman y Brownell, 2002; Pascual et al., 2011). Respecto a la ansiedad, Buckner, Silgado y Lewinsohn (2010) concluyen que la ansiedad es uno de los factores de riesgo de los TCA y Davey y Chapman (2009) la consideran como un rasgo permanente en muchas de las personas con TCA.

La bibliografía revisada nos conduce a plantearnos si las relaciones encontradas con poblaciones clínicas se dan también en adolescentes con alto riesgo de desarrollar un TCA, pero que no han llegado a desarrollarlo todavía. Este conocimiento ayudaría en el diseño de estrategias de prevención más adecuadas y específicas orientadas a esta población- diana. En este sentido, los objetivos del presente trabajo son los siguientes: a) estimar la prevalencia del riesgo de desarrollar un TCA en una muestra de adolescentes de 16 a 20 años; b) comprobar si hay diferencias entre las adolescentes de alto y bajo riesgo en la percepción de tener un problema de alimentación, en la percepción de su imagen corporal y en la sobrevaloración de la delgadez; c) analizar si hay diferencias entre los grupos de bajo y alto riesgo de desarrollar un TCA en el consumo de tabaco, alcohol y drogas ilegales; y d) comprobar si las adolescentes de alto riesgo presentan mayores niveles de ansiedad y alexitimia que las del grupo de bajo riesgo.

\section{Método}

\section{Tipo de estudio}

Esta investigación se puede calificar como un estudio ex post facto, con una muestra no probabilística.

\section{Participantes}

En el estudio participaron 767 mujeres, de 16 a 20 años, procedentes de 13 Institutos de Educación Secundaria y 5 centros universitarios de Guipúzcoa y Navarra. La edad media fue de 17.54 años y la desviación típica de 1.26. El 42.8\% de las estudiantes eran de $1^{\circ}$ de Bachillerato, el $19.2^{\%}$ de $2^{\circ}$ de Bachillerato, el $24.1^{\circ}$ cursaban $1^{\circ}$ de carrera y el $13.9^{\circ} \%$ estaban en $2^{\circ}$ de carrera. La evaluación se realizó durante los años 2007 y 2008. Las características demográficas y antropométricas de la muestra figuran descritas en la Tabla 1.

Tabla 1. Características demográficas y antropométricas de las participantes.

\begin{tabular}{lll}
\hline Variables demográficas y antropométricas & Muestra $(N=767)$ \\
\hline & $M$ & $D T$ \\
Edad & 17.54 & 1.26 \\
Peso (Kg.) & 59.97 & 9.19 \\
Altura (cm.) & 164 & 0.06 \\
Índice de Masa Corporal & 22.35 & 3.06 \\
& & \\
Curso & $n$ & $\%$ \\
$1^{\circ}$ de Bachillerato & 329 & 42.8 \\
$2^{\text {o de Bachillerato }}$ & 149 & 19.2 \\
$1^{\circ}$ de Carrera & 189 & 24.1 \\
$2^{\circ}$ de Carrera & 100 & 13.9 \\
& & \\
Ocupación del padre & $n$ & $\%$ \\
Empresarios y directivos & 102 & 13.2 \\
Profesionales & 122 & 15.9 \\
Técnicos y mandos intermedios & 89 & 11.7 \\
Empleados y obreros & 454 & 59.2 \\
\hline
\end{tabular}

\section{Instrumentos}

A las participantes se les administraron dos tipos de instrumentos, unos para formar los grupos de comparación 
(grupo de alto riesgo de desarrollar un TCA y grupo de bajo riesgo) y otros para evaluar las variables objeto de estudio.

Los instrumentos para formar los grupos están basados en los criterios utilizados por Gandarillas y Febrel (2000; véase apéndice 1). Junto al Índice de Masa Corporal y las Escalas de Obsesión por la Delgadez, Bulimia e Insatisfacción Corporal del Eating Disorder Inventory-2 (Garner, 1991; versión española de TEA, 1998), se formularon varias preguntas relativas al peso, la práctica del ejercicio físico y la utilización de conductas inadecuadas dirigidas a la pérdida de peso.

A su vez, se utilizaron los siguientes instrumentos para evaluar las variables objeto de estudio:

Cuestionario sociodemográfico. Este cuestionario se utilizó para recabar información sobre la edad, el nivel de escolaridad y la ocupación del padre.

Percepción de problema de alimentación. Se les realizó una pregunta referida a si creían que tenían un problema con la alimentación. Esta pregunta tenía 2 opciones de respuesta: 0 (no) y 1 (sî).

Percepción de la imagen corporal. Se evaluó a través de la siguiente pregunta: ¿Cómo te ves a ti misma? Las opciones de respuesta fueron: 1 (muy delgada), 2 (algo delgada), 3 (normal), 4 (gorda), 5 (algo gorda) y 6 (muy gorda).

Sobrevaloración de la imagen corporal. La evaluación se realizó a través de 3 preguntas con respuesta dicotómica (no $=0$ y sí = 1): ¿Crees que estar delgada es muy importante para encontrarte bien contigo misma?, ¿Crees que estar delgada es muy importante para que las cosas te vayan bien? y ¿Crees que estar delgada es muy importante para que los demás te valoren positivamente?

Consumo de sustancias adictivas. Para evaluar el consumo de tabaco, alcohol y otras drogas se seleccionaron 5 ítems de los recogidos en el Sistema de Vigilancia de Factores de Riesgo asociados a Enfermedades no Transmisibles de la Comunidad de Madrid (Consejería de Sanidad, 2002). Para el consumo de tabaco se realizaron dos preguntas, una referida al tipo de consumo $(0=$ no fuma, $1=$ alguna vez a la semana, $y$ $2=$ diariamente) y la otra al número de cigarrillos que fuma diariamente $(1=$ menos de $5,2=$ entre 5 y 10 , y $3=$ más de 10). En cuanto al consumo de alcohol, se formularon también dos preguntas: edad de comienzo de ingesta de alcohol al menos una vez por semana $(0=$ nunca, $1=$ entre 11 y 12 años, $2=$ entre 13 y 14; $3=$ entre 15 y 16, y $4=$ más de 16 años) y días que ha tomado una bebida alcohólica durante el último mes $(0=$ ninguno, $1=$ entre 1 y 5 días, $2=$ entre 6 y 9 , y $3=$ más de 10 días). La quinta pregunta explora si alguna vez se han consumido algunas de las siguientes sustancias: tranquilizantes o pastillas para dormir sin prescripción médica, cannabis, cocaína, heroína y otras drogas no incluidas en la lista. Las opciones de respuesta para cada sustancia fueron: 0 (nunca) y 1 (alguna vez).

Escala de Ansiedad-Rasgo del Cuestionario de Ansiedad EstadoRasgo (STAI) (Spielberger, Gorsuch y Lushene, 1970; versión española de TEA, 1994). Esta escala está compuesta por 20 ítems. A las participantes se les pide que valoren cómo se sienten en general habitualmente y las opciones de respuesta de cada ítem se presentan en una escala de tipo Likert que oscila de 0 (casi nunca) a 3 (casi siempre). El rango de puntuaciones oscila de 0 a 60 . El coeficiente de consistencia interna (alfa de Cronbach) obtenido en la escala fue de .91.

Escala de Alexitimia de Toronto (TAS-20) (Bagby, Parker y Taylor, 1994; versión española de Martínez-Sánchez, 1996). Se aplicó una versión abreviada (de 12 ítems) de esta escala. Dichos ítems están agrupados en dos subescalas: Dificultad para identificar las emociones y Dificultad para expresar las emociones. A las participantes se les pedía que indicasen en una escala de 5 puntos en qué medida estaban de acuerdo o en desacuerdo con cada uno de los ítems: 1 (totalmente en desacuerdo) y 5 (totalmente de acuerdo). Los coeficientes de consistencia interna (alfa de Cronbach) obtenidos fueron los siguientes: .88 en la primera subescala y .80 en la segunda.

\section{Procedimiento}

A las participantes se les entregaron los cuestionarios con las tareas que debían realizar y las instrucciones para llevarlas a cabo. Tenían aproximadamente media hora para rellenar las escalas y cuestionarios y firmar una hoja de consentimiento informado cuando eran mayores de edad. En los casos en los que la chica era menor de edad, se solicitó el consentimiento informado de la familia. En todos los casos se les recordaba que la información solicitada era anónima y confidencial.

La aplicación de los instrumentos fue realizada por dos psicólogas en las propias aulas de los centros escolares o universitarios. Aunque las pruebas se aplicaron al total de la clase, para este estudio únicamente se ha tenido en cuenta a la población femenina. Una vez cumplimentados los cuestionarios, una de las psicólogas medía el peso y la altura de cada participante para obtener el IMC. Se les ofreció la devolución de los resultados para motivar su participación en el estudio.

\section{Análisis estadístico}

Los análisis estadísticos han sido llevados a cabo con el programa SPSS (versión 19.0 para Windows). Para analizar las diferencias entre el grupo de control y el grupo de riesgo de TCA se han realizado análisis de carácter descriptivo (porcentajes, medias y desviaciones típicas) y se han calculado distintas pruebas de significación en función de que las variables fueran cuantitativas o cualitativas $\left(t\right.$ de Student y $\chi^{2}$ -chi cuadrado-, respectivamente), así como las pruebas del tamaño del efecto correspondientes ( $d$ de Cohen y $V$ de Cramer, respectivamente).

\section{Resultados}

De las 767 adolescentes que participaron en el estudio, 651 $(84.9 \%)$ no presentaban conductas de riesgo de TCA (grupo de bajo riesgo) y $116(15.1 \%)$ presentaban conductas indicadoras de un alto riesgo de TCA (grupo de alto riesgo), según los criterios recogidos en el apéndice 1. En concreto, un $1.7 \%$ cumplían criterios para posible riesgo de anorexia, un 
$10.7 \%$ para posible riesgo de purga/atracón y un $2.7 \%$ cumplían criterios para ambos tipos de riesgo.

\section{Variables sociodemográficas y antropométricas}

En cuanto a las variables sociodemográficas estudiadas, únicamente se observaron diferencias estadísticamente significativas entre el grupo de bajo y el grupo de alto riesgo de TCA en la variable ocupación del padre, $\chi^{2}(3, N=767)=$ $16.96, p<.001$. Aunque la magnitud de la diferencia es muy pequeña $(V=.16)$, un porcentaje mayor de adolescentes de alto riesgo tenían padres profesionales, mientras que en el grupo de bajo riesgo un mayor porcentaje de padres se halla- ban en las categorías de técnicos y mandos intermedios o empleados y obreros. En el resto de las variables (edad y curso) no se hallaron diferencias entre ambos grupos.

En relación con las variables antropométricas, se encontraron diferencias significativas en el peso, $t(1,765)=7.2, p$ $<.001, d=0.83$, $y$ en el IMC, $t(1,765)=7.9, p<.001, d=$ 1.18 , siendo el grupo de alto riesgo de TCA el que presentaba mayor peso e IMC. Como se puede observar, en ambas variables la magnitud de las diferencias fue alta. A su vez, en las variables relativas a la edad de la menarquia, el peso deseado y la altura no se observaron diferencias significativas entre ambos grupos (Tabla 2).

Tabla 2. Diferencias entre los grupos con bajo y alto de riesgo de TCA en variables sociodemográficas y antropométricas.

\begin{tabular}{|c|c|c|c|c|c|}
\hline \multirow[t]{2}{*}{$\overline{\text { Variables }}$} & \multicolumn{2}{|c|}{ Bajo riesgo de TCA $(n=651)$} & \multicolumn{2}{|c|}{ Alto riesgo de TCA $(n=116)$} & \multirow[t]{2}{*}{$t / \chi^{2}$} \\
\hline & $M$ & $D T$ & $M$ & $D T$ & \\
\hline$\overline{E d a d}$ & 17.56 & 1.3 & 17.43 & 1.3 & $t=1.0$ \\
\hline Edad de la menarquía & 12.7 & 1.2 & 12.4 & 1.4 & $t=2.1$ \\
\hline Peso (Kg.) & 58.78 & 8.2 & 66.7 & 11.4 & $t=7.2^{* * *}$ \\
\hline Peso deseado (Kg.) & 54.67 & 5.6 & 55.75 & 5.3 & $t=1.8$ \\
\hline Altura (cm.) & 164 & 0.06 & 163 & 0.06 & $t=0.3$ \\
\hline Índice de Masa Corporal & 21.89 & 2.6 & 24.9 & 3.9 & $t=7.9^{* * *}$ \\
\hline Curso & $N$ & $\%$ & $n$ & $\%$ & \\
\hline $1^{\circ}$ de Bachillerato & 272 & 42.2 & 57 & 49.2 & $\chi^{2}=6.9$ \\
\hline $2^{\circ}$ de Bachillerato & 121 & 18.5 & 28 & 24.1 & \\
\hline $1^{\circ}$ de Carrera & 168 & 25.5 & 21 & 18.1 & \\
\hline $2^{\circ}$ de Carrera & 90 & 13.5 & 10 & 8.6 & \\
\hline \multicolumn{6}{|l|}{ Ocupación del padre } \\
\hline Empresarios y directivos & 86 & 13.1 & 16 & 13.5 & $\chi^{2}=16.96^{* *}$ \\
\hline Profesionales & 89 & 13.6 & 33 & 28.8 & \\
\hline Técnicos y mandos intermedios & 81 & 12.6 & 8 & 6.7 & \\
\hline Empleados y obreros & 395 & 60.7 & 59 & 51.2 & \\
\hline
\end{tabular}

Empleados y obreros

Percepción del problema, percepción de la imagen corporal y sobrevaloración de la delgadez

En cuanto a la percepción del problema, un $32 \%$ de las adolescentes del grupo de alto riesgo de TCA (frente al 0\% de las del grupo de bajo riesgo) pensaba que tenía dificultades con la alimentación. La diferencia entre ambos grupos fue estadísticamente significativa, $\chi^{2}(1, N=766)=220.08, p$ $<.001$, y de magnitud moderada $(V=.56)$.

Respecto a la percepción de la imagen corporal, ninguna de las adolescentes del grupo de alto riesgo de TCA (frente al $9.6 \%$ de las del grupo de bajo riesgo) creía que estaba algo o muy delgada y el $82.6 \%$ (frente al $29.1 \%$ de las del grupo de bajo riesgo) creía que estaba algo o muy gorda. También en esta variable se presentaron diferencias significativas moderadas entre ambos grupos, $\chi^{2}(4, N=766)=195.65, p<$ $.001, V=.51$.

En relación con la sobrevaloración de la delgadez, un mayor número de adolescentes del grupo de alto riesgo pensaba que la delgadez es muy importante para estar satisfecha con una misma, $\chi^{2}(1, N=766)=63.15, p<.001, V=.29$, para que las cosas te vayan bien, $\chi^{2}(1, N=766)=70.33, p<$ $.001, V=.30$, y para que los demás te valoren positivamente $\chi^{2}(1, N=766)=74.05, p<.001, V=.31$ (Tabla 3). En todos estos casos las diferencias fueron significativas, si bien la magnitud de las mismas no era alta. 
Tabla 3. Diferencias entre los grupos de bajo y alto riesgo de TCA en percepción de problema, percepción de la imagen corporal y sobrevaloración de la delgadez.

\begin{tabular}{|c|c|c|c|c|c|}
\hline \multirow[t]{2}{*}{ Variables } & \multicolumn{2}{|c|}{ Bajo riesgo de TCA $(n=651)$} & \multicolumn{2}{|c|}{ Alto riesgo de TCA $(n=115)$} & \multirow[t]{2}{*}{$\chi^{2}$} \\
\hline & $n$ & $\%$ & $n$ & $\%$ & \\
\hline \multicolumn{6}{|c|}{ ¿Crees qué tienes problemas con la alimentación? } \\
\hline Sí & 0 & 0 & 37 & 32.2 & $220.08^{* * *}$ \\
\hline No & 651 & 100 & 78 & 67.8 & \\
\hline \multicolumn{6}{|c|}{ ¿Cómo te ves a ti misma? } \\
\hline Muy gorda & 3 & 0.5 & 25 & 21.7 & $195.65^{* * *}$ \\
\hline Algo gorda & 186 & 28.6 & 70 & 60.9 & \\
\hline Normal & 399 & 61.3 & 20 & 17.4 & \\
\hline Algo delgada & 52 & 7.9 & 0 & 0 & \\
\hline Muy delgada & 11 & 1.7 & 0 & 0 & \\
\hline \multicolumn{6}{|c|}{ Estar delgada es muy importante: } \\
\hline \multicolumn{6}{|c|}{ Para encontrarte bien contigo misma } \\
\hline Sí & 434 & 66.6 & 31 & 27.2 & $63.15^{* * *}$ \\
\hline No & 217 & 33.4 & 84 & 72.8 & \\
\hline \multicolumn{6}{|c|}{ Para que las cosas te vayan bien } \\
\hline Sí & 596 & 91.6 & 73 & 63.1 & $70.33^{* * *}$ \\
\hline No & 55 & 8.4 & 42 & 36.9 & \\
\hline \multicolumn{6}{|c|}{ Para que los demás te valoren positivamente } \\
\hline Sí & 592 & 91 & 70 & 60.6 & $74.05^{* * *}$ \\
\hline No & 59 & 9 & 45 & 39.4 & \\
\hline
\end{tabular}

\section{Consumo de sustancias adictivas}

Un porcentaje significativamente mayor de adolescentes del grupo de alto riesgo de TCA fumaba, bien sea alguna vez a la semana $(21.5 \%$ frente al $14.1 \%$ del grupo de bajo riesgo) o diariamente $(34.5 \%$ frente al $26.7 \%)$. La diferencia entre ambos grupos en la frecuencia del consumo de tabaco fue significativa, pero de baja magnitud $\chi^{2}(2, N=766)=9.75, p$ $<.01, V=.11$. Sin embargo, no aparecían diferencias entre ambos grupos en la cantidad de cigarrillos que fumaban.
Respecto al consumo de alcohol, en las adolescentes del grupo de alto riesgo había un mayor porcentaje de chicas que empezaron a consumir entre 11 y 14 años (45.7\%) que en el grupo de bajo riesgo (37.5\%). Esta diferencia en la edad en que empezaron a consumir alcohol semanalmente fue significativa, pero de baja magnitud, $\chi^{2}(4, N=766)=9.65, p<$ $.05, V=.11$. Sin embargo, no se obtuvieron diferencias entre los grupos en el número de días que habían consumido alcohol durante el último mes (Tabla 4).

Tabla 4. Diferencias entre los grupos de bajo y alto riesgo de TCA en el consumo de tabaco y alcohol.

\begin{tabular}{|c|c|c|c|c|c|}
\hline \multirow[t]{2}{*}{ Consumo de tabaco y alcohol } & \multicolumn{2}{|c|}{ Bajo riesgo de TCA $(n=650)$} & \multicolumn{2}{|c|}{ Alto riesgo de TCA $(n=116)$} & \multirow[t]{2}{*}{$\chi^{2}$} \\
\hline & $n$ & $\%$ & $n$ & $\%$ & \\
\hline \multicolumn{6}{|l|}{ Consumo de tabaco } \\
\hline No fuma & 385 & 59.2 & 51 & 44 & $9.75^{* *}$ \\
\hline Alguna vez a la semana & 92 & 14.1 & 25 & 21.5 & \\
\hline Diariamente & 173 & 26.7 & 40 & 34.5 & \\
\hline \multicolumn{6}{|l|}{ Numero de cigarrillos } \\
\hline Menos de 5 & 88 & 34 & 20 & 31.3 & 0.478 \\
\hline Entre 6 y 10 & 120 & 46.3 & 29 & 45.3 & \\
\hline Más de 10 & 51 & 19.7 & 15 & 23.4 & \\
\hline \multicolumn{6}{|l|}{ Consumo de alcohol } \\
\hline \multicolumn{6}{|l|}{ Edad empezó a consumir semanalmente } \\
\hline Nunca ha consumido con esa frecuencia & 142 & 21.8 & 21 & 18.1 & $9.65^{*}$ \\
\hline Entre 11 y 12 años & 19 & 2.9 & 8 & 6.9 & \\
\hline Ente 13 y 14 años & 225 & 34.6 & 45 & 38.8 & \\
\hline Entre 15 y 16 & 217 & 33.5 & 29 & 25 & \\
\hline Mayor de 16 & 47 & 7.3 & 13 & 11.2 & \\
\hline \multicolumn{6}{|l|}{ Dias de consumo durante el último mes } \\
\hline Ninguno & 82 & 12.6 & 10 & 8.6 & 5.81 \\
\hline Entre 1 y 5 & 470 & 72.3 & 91 & 78.4 & \\
\hline Entre 6 y 9 & 81 & 12.5 & 9 & 7.8 & \\
\hline Más de 10 & 17 & 2.6 & 6 & 5.2 & \\
\hline
\end{tabular}

${ }^{*} p<.05 ; * * p<.01$ 
En cuanto al consumo de otras drogas, no se hallaron diferencias significativas entre uno y otro grupo en el consumo de tranquilizantes o pastillas para dormir sin receta ni en el consumo de cannabis, cocaína o heroína. A su vez, un 18\% de las adolescentes con alto riesgo de TCA (frente a un
$10.7 \%$ de las del grupo de bajo riesgo) informó haber consumido alguna vez otras drogas. Fue la única variable en la que se encontró una diferencia significativa, $\chi^{2}(1, N=766)$ $=4.81, p<.05, V=.08$, aunque muy pequeña (Tabla 5).

Tabla 5. Diferencias entre los grupos de bajo y alto riesgo de TCA en el consumo de drogas.

\begin{tabular}{|c|c|c|c|c|c|}
\hline \multirow[t]{2}{*}{ Consumo de drogas } & \multicolumn{2}{|c|}{ Bajo riesgo de TCA $(n=650)$} & \multicolumn{2}{|c|}{ Alto riesgo de TCA $(n=116)$} & \multirow[t]{2}{*}{$\chi^{2}$} \\
\hline & $n$ & $\%$ & $n$ & $\%$ & \\
\hline \multicolumn{6}{|c|}{ Tranquilizantes o pastillas sin receta } \\
\hline Nunca & 572 & 87.9 & 98 & 84.5 & 0.97 \\
\hline Alguna vez & 79 & 12.1 & 18 & 15.5 & \\
\hline \multicolumn{6}{|l|}{ Cannabis } \\
\hline Nunca & 188 & 28.9 & 24 & 21.1 & 2.99 \\
\hline Alguna vez & 463 & 71.1 & 92 & 78.9 & \\
\hline \multicolumn{6}{|l|}{ Cocaína } \\
\hline Nunca & 594 & 91.3 & 106 & 91 & 0.013 \\
\hline Alguna vez & 57 & 8.7 & 10 & 9 & \\
\hline \multicolumn{6}{|l|}{ Heroina } \\
\hline Nunca & 648 & 99.5 & 116 & 100 & 0.533 \\
\hline Alguna vez & 3 & 0.5 & 0 & 0 & \\
\hline \multicolumn{6}{|l|}{ Otras drogas } \\
\hline Nunca & 581 & 89.3 & 95 & 82 & $4.81^{*}$ \\
\hline Alguna vez & 70 & 10.7 & 21 & 18 & \\
\hline
\end{tabular}

\section{Ansiedad y alexitimia}

Las adolescentes del grupo de alto riesgo de TCA mostraban niveles de ansiedad-rasgo significativamente más altos que las del grupo de bajo riesgo, $t(1,759)=10.17, p<.001$, $d=0.99$. Respecto a la alexitimia, las adolescentes del grupo de alto riesgo presentaban niveles de dificultad marcadamente más altos tanto en la identificación de sentimientos, $t(1$, $764)=5.61, p<.001, d=0.72$, como en la expresión de los mismos, $t(1,764)=3.65, p<.001, d=0.5$ (tabla 6). En todos los casos la magnitud de las diferencias fue considerable.

Tabla 6. Diferencias entre lo grupos de bajo y alto riesgo de TCA en ansiedad-rasgo y alexitimia

\begin{tabular}{|c|c|c|c|c|c|}
\hline \multirow[t]{2}{*}{ Variables } & \multicolumn{2}{|c|}{ Bajo riesgo de TCA $(n=650)$} & \multicolumn{2}{|c|}{ Alto riesgo de TCA $(n=116)$} & \multirow[t]{2}{*}{$t$} \\
\hline & $M$ & $D T$ & $M$ & $D T$ & \\
\hline Ansiedad rasgo & 21.68 & 7.8 & 29.97 & 9.2 & $-10.17 * * *$ \\
\hline Alexitimia & 30.82 & 8.5 & 35.89 & 9.6 & $-5.34 * * *$ \\
\hline Dificultad identificar sentimientos & 16.98 & 5.2 & 20.45 & 6.3 & $-5.61 * * *$ \\
\hline Dificultad expresar sentimientos & 13.84 & 4.3 & 15.44 & 4.3 & $-3.65^{* * *}$ \\
\hline
\end{tabular}
$* * * p<.001$

\section{Discusión}

En la muestra estudiada la prevalencia de chicas adolescentes con alto riesgo de desarrollar un TCA es del 15.1\%. Este dato es similar al obtenido en otros estudios realizados con mujeres de esta edad (Ballester, de Gracia, Patiño, Suñol y Ferrer, 2002; Gandarillas y Febrel, 2000; Sepúlveda et al., 2008; Quiles-Marcos et al., 2011; Toro et al., 2006). De este 15\% de adolescentes en posible riesgo de TCA, un 1.7\% cumplía criterios para posible riesgo de anorexia, un $10.7 \%$ para posible riesgo de bulimia (purga/atracón) y un $2.7 \%$ para ambos tipos de riesgo. Mientras que la tasa del $1.7 \%$ obtenida para el grupo con posible anorexia resulta congruente, dado que la prevalencia hallada en estudios previos la sitúan entre el $0.3 \%$ y el $2.3 \%$, no sucede lo mismo con la tasa del $10.7 \%$ en posible riesgo de bulimia, ya que los estudios epidemiológicos sitúan su prevalencia entre el $0.44 \%$ y el $2.29 \%$ (Olesti et al., 2008; Peláez-Fernández, Raich y Labrador, 2010; Toro et al., 2006). Una posible explicación del alto porcentaje de adolescentes con riesgo de bulimia en esta muestra es que en los criterios establecidos para riesgo de purga/atracón no se han tenido en cuenta la frecuencia y duración de las conductas de control del peso (vómitos, laxantes o diuréticos) que establece el DSM-IV-TR, porque únicamente se preguntaba si se habían realizado alguna vez durante el último año. Pero el objetivo de nuestro estudio era determinar el riesgo de TCA (no el TCA propiamente dicho), por lo que no resulta incongruente que las tasas resulten más altas que en los estu- 
dios epidemiológicos de tipo puramente clínico. En cualquier caso, el alto porcentaje obtenido evidencia la necesidad de diseñar estrategias de prevención de conductas bulímicas en las adolescentes.

Las variables sociodemográficas relativas a la edad y el curso no resultan relevantes en este estudio para diferenciar entre adolescentes con alto y bajo riesgo de desarrollar un TCA, pero sí que hay diferencias en función de la ocupación del padre. Al contrario de lo hallado en otros estudios (González-Juárez et al., 2007; Sepúlveda et al., 2008), una proporción superior de adolescentes de alto riesgo de TCA tienen padres con profesiones liberales, lo que denota su pertenencia a una clase social media o media-alta.

En cuanto a las variables antropométricas, el peso y el IMC resultan ser factores muy relevantes para el riesgo de desarrollar un TCA. Así, las adolescentes de alto riesgo tienen de media 8 kilos más que las adolescentes del grupo de bajo riesgo. Mientras estas últimas tienen un IMC de normopeso $($ IMC medio $=21.89)$, las del grupo de alto riesgo presentan un IMC medio cercano al sobrepeso (IMC medio = 24.9). En consonancia con estudios previos (Babio, Canals, Pietrobelli, Pérez y Arija, 2009; Gasco et al., 2010; Veses et al., 2010), el sobrepeso o la obesidad se configuran como un factor de riesgo importante para el desarrollo de los TCA. No obstante, la percepción que se tiene del propio cuerpo es tan importante o más que el peso real (Cruz y Maganto, 2002; Ramos, Rivera de los Santos y Moreno, 2010). Así, el $59.6 \%$ de las adolescentes de alto riesgo de TCA de nuestro estudio tiene un IMC de normopeso y el $40.4 \%$ muestra un cierto sobrepeso u obesidad. Sin embargo, solo el $17.4 \%$ se percibe con un peso normal, mientras que el $82.6 \%$ restante se percibe como algo o muy gorda. Todo ello pone de manifiesto la importancia de promover programas preventivos para adolescentes orientados a la aceptación de su imagen corporal, a fin de evitar la implicación en conductas inadecuadas de control del peso que puedan desencadenar trastornos alimentarios.

Además, a los factores anteriores hay que añadir una mayor sobrevaloración de la delgadez en las adolescentes de alto riesgo. Esta sobrevaloración supone una mayor interiorización del ideal estético corporal (delgadez), que no se limita a los estándares de belleza, sino que se le asigna un valor fundamental en la autoestima, ya que está ligada a la satisfacción personal, el éxito y la aceptación social, tal como es señalado también por Dittmar (2008). Por ello, la investigación futura sobre adolescentes en riesgo de TCA debe prestar atención en mayor profundidad al impacto de los ideales culturales vigentes en el proceso de construcción de la identidad personal.

En cuanto a la relación entre el riesgo de TCA y el consumo de sustancias en adolescentes con alto riesgo de desarrollar un TCA, los resultados obtenidos muestran, como en otros estudios (Bisetto et al., 2012; Cabrera, Toledo y Báez, 2006), escasas relaciones entre ambos aspectos. En relación al consumo de tabaco, si bien un mayor número de adolescentes con alto riesgo de TCA fuman, no hay, sin embargo, diferencias en la cantidad de cigarrillos, a diferencia de los datos de Quiles-Marcos et al., (2012). A su vez, las adolescentes con mayor riesgo de TCA comienzan a consumir alcohol a una edad más temprana (como en el estudio de Herrero et al., 2010), pero no se encuentran diferencias en el número de días que se ha consumido alcohol durante el último mes (como en el estudio de Bisetto et al., 2012). Por lo que se refiere al consumo de drogas, la única diferencia encontrada es que un mayor porcentaje de las adolescentes de alto riesgo de TCA han consumido alguna vez drogas de síntesis. Esta falta de relación entre consumo de drogas ilegales (cannabis, heroína o cocaína) y riesgo de TCA también es encontrada por unos autores (Bisetto et al., 2012; QuilesMarcos et al., 2012), pero no por otros (Herrero et al., 2010). La falta de claridad de esta relación puede tener que ver con que la evaluación del consumo de sustancias ilegales en términos exclusivamente dicotómicos (consumo-no consumo) resulte poco útil para valorar las relaciones entre riesgo de TCA y consumo de sustancias. La falta de datos sobre cantidad, frecuencia y tiempo de consumo imposibilita que se puedan tener indicadores que permitan diferenciar niveles de consumo que suponen un mayor riesgo. Esto constituye una limitación del estudio que nos proponemos subsanar en futuros trabajos. Cabe asimismo plantearse si el mayor número de adolescentes con alto riesgo de TCA que consumen sustancias adictivas (tabaco y drogas de síntesis) corresponde a una multiimpulsividad como rasgo de personalidad o es una estrategia de afrontamiento para superar el malestar emocional derivado de una insatisfacción con la imagen corporal. Esta cuestión requiere nuevos estudios con diferente metodología.

Finalmente, los resultados obtenidos en relación con las variables emocionales alexitimia y ansiedad son consistentes con los hallados en otros estudios con muestras de riesgo y clínicas. Así, las adolescentes en riesgo de TCA presentan niveles mucho más altos de alexitimia, especialmente en la identificación de emociones (Iancu, Cohen, Yehuda y Kotler, 2006; Pascual et al., 2011; Ridout, Thom y Wallis, 2010), asî como de ansiedad-rasgo (Buckner et al., 2010; Davey y Chapman, 2009; Marañón, Grijalvo y Echeburúa, 2007; Pascual et al., 2011). Una posible hipótesis explicativa es que, en las adolescentes con alto riesgo de TCA, las dificultades en la identificación y expresión de emociones ligadas a una alta ansiedad favorecen un estilo cognitivo dirigido hacia lo externo y concreto, en el que predominan las respuestas fisiológicas y conductuales, con menoscabo en la regulación emocional (Taylor y Bagby, 2000; Taylor, Bagby y Parker, 1997). Para la comprobación de esta hipótesis sería necesario llevar a cabo un estudio longitudinal.

El interés de este estudio deriva de sus posibles aplicaciones prácticas. Así, los programas para la prevención de los trastornos alimentarios deberían incluir, además de educación nutricional, información sobre el efecto nocivo de la interiorización del ideal de delgadez extrema imperante, así como técnicas de expresión y regulación emocional, a fin de que no tengan que recurrir a conductas inadecuadas de con- 
trol del peso y de la imagen corporal (entre estas, el consumo de tabaco y de otras drogas).

Finalmente, este estudio presenta algunas limitaciones. Al tratarse de un estudio transversal, no se puede inferir causalidad ni es posible tampoco generalizar los resultados por el método de selección muestral utilizado. En cuanto a la investigación futura, sería conveniente realizar un estudio de do- ble fase con entrevista diagnóstica (clínica), así como incorporar sujetos de ambos sexos.

Agradecimientos.- Este artículo ha sido apoyado por el Grupo Consolidado de Investigación en Psicología Clínica del Gobierno Vasco y por la UFI 11/04 de la Universidad del País Vasco (UPV/EHU).

\section{Referencias}

American Psychiatric Association (2000). Diagnostic and Statistical Manual of Mental Disorders (4th ed.Test Revised). Washington, D. C.: American Psychiatric Association.

Anzengruber, D., Klump, K. L., Thornton, L., Brandt, H., Crawford, S., Fitcher, M. M. et al. (2006). Smoking in eating disorders. Eating Behaviors, 7, 291-299.

Babio, N., Canals, J., Pietrobelli, A., Pérez, S. y Arija V. (2009). A two-phase population study: relationships between overweight, body composition and risk of eating disorders. Nutrición Hospitalaria, 24, 485-91.

Bagby, R. M., Parker, J. D. A. y Taylor, G. J. (1994). The twenty-item Toronto Alexithymia Scale-I: Item selection and cross-validation of the factor structure. Journal of Psychosomatic Research, 38, 23-32.

Ballester, D., de Gracia, M., Patiño, J., Suñol, C. y Ferrer, M. (2002). Actitudes alimentarias y satisfacción corporal en adolescentes: un estudio de prevalencia. Actas Españolas de Psiquiatría, 30, 207-212.

Bisetto, D., Botella, A. y Sancho, A. (2012). Trastornos de la conducta alimentaria y consumo de drogas en población adolescente. Adicciones, 24, 9-16.

Bruch, H. (1962). Perceptual and conceptual disturbances in anorexia nerviosa. Psychosomatic Medicine, 24, 187-194.

Bruch, H. (1981). Developmental considerations of anorexia nervosa and obesity. Canadian Journal of Psychiatry, 26, 212-217.

Buckner, J. D., Silgado, J. y Lewinsohn, P. M. (2010). Delineation of differential temporal relations between specific eating and anxiety disorders. Journal of Psychiatric Research, 44, 781-787.

Calero-Elvira, A., Krug, F., Dabis, K., López, C., Fernández-Aranda, F. y Treasure, J. (2009). Meta-analysis on drugs in people with eating disorders. European Eating Disorders Review, 17, 243-259.

Cabrera, J. L., Toledo, I. y Báez, A. (2006). Riesgo de trastornos del comportamiento alimentario en adolescentes de un área de salud. Semergen, 32, 258-264.

Consejería de Sanidad (2002). Hábitos de salud en la población juvenil de la Comunidad de Madrid. Sistema de Vigilancia de Factores de Riesgo Asociados a enfermedades no Transmisibles. Boletín Epidemiológico de la Comunidad de Madrid, 8, 3-42.

Crow, S., Eisenberg, M., Story, M. y Neumark-Sztainer, D. (2006). Psychosocial and behavioral correlates of dieting among overweight and non-overweight adolescents. Journal of Adolescent Health, 38, 569-574.

Cruz, S. y Maganto, C. (2002). Los trastornos de la imagen corporal y los trastornos alimenticios en adolescentes: Un estudio empírico. Psiquis, 23, 36-43.

Davey, G. C. L. y Chapman, L. (2009). Disgust and eating disorder symptomatology in a non-clinical population: The role of trait anxiety and anxiety sensitivity. Clinical Psychology and Psychotherapy, 16, 268-275.

Dittmar, H. (2008). Consumer culture, identity and wellbeing: The search for the 'Good Life' and the 'Body Perfect'. Sussex: Psychology Press.

Espinoza, P., Penelo, E. y Raich, R. M. (2010). Disordered eating behaviors and body image in a longitudinal pilot study of adolescent girls: What happens 2 years later? Body Image, 7, 70-73.

Gadalla, T. y Piran, N. (2007). Eating disorders and substance abuse in Canadian men and women: a national study. Eating Disorders, 15, 189-203.

Gandarillas, A. y Febrel, C. (2000). Encuesta de prevalencia de trastornos del comportamiento alimentario en adolescentes escolarizados de la Comunidad de Madrid. Madrid: Consejería de Sanidad.

Garner, D. M. (1991). Eating Disorder Inventory-2: Professional manual. Odessa, Florida: Psychological Assessment Resources (versión española, TEA, 1998).
Gasco, M., Briñol, P. y Horcajo, J. (2010). Cambio de actitudes hacia la imagen corporal: El efecto de la elaboración sobre la fuerza de las actitudes. Psicothema, 22, 77-82.

Gilboa-Schechtman, E., Avnon, L., Zubery, E. y Jeczmien, P. (2006). Emotional processing in eating disorders: Specific impairment or general distress related deficiency? Depression and Anxiety, 23, 331- 339.

González, M., Penelo, E., Gutiérrez, T. y Raich, R. M. (2010). Seguimiento a los 30 meses de un programa de prevención: el IMC como predictor de sintomatomatología alimentaria en chicas escolarizadas. Cuadernos de Medicina Psicosomática, 95,35-43.

González-Juárez, C., Pérez-Pérez, E., Martín Cabrera, B., Mitja Pau, I., Roy de Pablo, R. y Vázquez de la Torre Escalera, P. (2007). Detection of adolescents at risk of suffering eating disorders. Atención Primaria, 39, 189-194.

Gutiérrez, R., Mora, J., Unikel, C., Villatoro, J. y Medina-Mora, M. E. (2001). El consumo de drogas en una muestra de mujeres estudiantes que presentan conductas alimentarias de riesgo. Salud Mental, 24, 55-61.

Hayaki, J. (2009). Negative reinforcement eating expectancies, emotion dysregulation, and symptoms of bulimia nervosa. International Journal of Eating Disorders, 42, 552-556.

Hayaki, J., Friedman, M. y Brownell, K. (2002). Emotional expression and body dissatisfaction. International Journal of Eating Disorders, 31, 57-62.

Herrero, M., Viña, C. y Ramírez, G. (2010). Eating attitudes and their relation with drug consumption in a university sample. The European Journalof Psychiary, 24, 9-17.

Hunot, C., Vizmanos, B., Vázquez-Garibay, E. M. y Celis, A. J. (2008). Definición conceptual de las alteraciones de la conducta alimentaria. Revista de Salud Pública y Nutrición 9(1). Recuperado de http://www.respyn.uanl.mx/ix/1/ensayos/alteraciones.htm

Iancu, I., Cohen, E., Yehuda, Y. B. y Kotler, M. (2006). Treatment of eating disorders improves eating symptoms but not alexithymia and dissociation proneness. Comprehensive Psychiatry, 47, 189-193.

Krahn, D., Kurth, C. L., Demitrack, M. y Drewnowski, A. (1992). The relationship of dieting severity and bulimic behaviors to alcohol and other drug use in young women. Journal of Substance Abuse, 4, 341-353.

Luce, K., Engler, P. y Crowther, J. (2007). Eating disorders and alcohol use: group differences in consumption rates and drinking motives. Eating Behaviors, 8, 177-184. doi:10.1016/j.eatbeh.2006.04.003

Marañón, I., Echeburúa, E. y Grijalvo, J. (2004). Prevalence of personality disorders in patients with eating disorders: An exploratory study. European Eating Disorders Review, 12, 217-222.

Marañón, I., Echeburúa, E. y Grijalvo, J. (2007). Are there more personality disorders in treatment-seeking patients with eating disorders than in other kind of psychiatric patients? A two control groups comparative study using the IPDE. International Journal of Clinical and Health Psychology, 7, 283-293.

Marañón, I., Grijalvo, J. y Echeburúa, E. (2007). Do the IPDE and the MCMI assess the same personality disorders in patients with eating disorders? International Journal of Clinical and Health Psychology, 7, 587-594.

Martínez-Sánchez, F. (1996). Adaptación española de la Escala de Alexitimia de Toronto (TAS-20). Clínica y Salud, 7, 19-32.

Neumark-Sztainer, D. R., Wall, M. M., Haines, J. I., Story, M. T., Sherwood, N. E. y Van den Berg, P. A. (2007). Shared risk and protective factors for overweight and disordered eating in adolescents. American Journal of Preventive Medicine, 33, 359-369.

Olesti, M., Piñol, J. L., Martín, N., de la Fuente, M., Riera, A., Bofarull, J. M., et al. (2008). Prevalencia de anorexia nerviosa, bulimia nerviosa y 
otros TCA en adolescentes femeninas de Reus. Anales de Pediatría, 68 (1), 18-23.

Overton, A., Selway, S., Strongman, K. y Houston, M. (2005). Eating disorders-The regulation of positive as negative emotion experience. Journal of Clinical Psychology in Medical Settings, 12, 39-56.

Pascual, A., Etxebarria, I., Cruz, M. S. y Echeburúa, E. (2011). Las variables emocionales como factores de riesgo de los trastornos de la conducta alimentaria. International Journal of Clinical and Health Psychology, 11, 229242.

Peláez-Fernández, M. A., Raich, R. M. y Labrador, F. J. (2010). Eating disorders in Spain: Revision of empirical epidemiological studies. Mexican Journal of Eating Disorders, 1, 62-75.

Quiles-Marcos, Y., Balaguer-Solá, I., Pamies-Aubalat, L., Quiles-Sebastián, M. J., Marzo-Campos, J. C. y Rodríguez-Marín, J. (2011). Eating Habits, Physical Activity, Consumption of Substances and Eating Disorders in Adolescents. The Spanish Journal of Psychology, 14, 712-723.

Ramos, P., Rivera de los Santos, F. y Moreno, C. (2010). Diferencias de sexo en imagen corporal, control de peso e índice de masa corporal de los adolescentes españoles. Psicothema, 22, 77-83.

Ridout, N., Thom, C. y Wallis, D. J. (2010). Emotion recognition and alexithymia in females with nonclinical disordered eating. Eating Behaviors, 11, 1-5.

Sancho, C., Arija, M. V., Asorey, O. y Canals, J. (2007). Epidemiology of eating disorders: A two-year follow-up in an early adolescent school population. European Child \& Adolescent Psychiatry, 16, 495-504.

Schmidt, U. y Treasure, J. (2006). Anorexia nervosa: Valued and visible. A cognitive-interpersonal maintance model and its implications for research and practice. British Journal of Clinical Psychology, 45, 343-366

Sepúlveda, A. R., Carrobles, J. A., y Gandarillas, A. (2008). Gender, school and academic year differences among Spanish university students at high-risk for developing an eating disorder: an epidemiologic study. BMC Public Health, 8, 1-12.
Stice, E. y Shaw, H. (2010). Risk factors and prodromal eating pathology. Journal of Child Psychology and Psychiatry, 51, 518-525.

Stice, E., Marti, C. N. Y Durant, S. (2011). Risk factors for onset of eating disorders: Evidence of multiple risk pathways from an 8-year prospective study. Behaviour Research and Therapy, 49, 622-627.

Spielberger, C. D., Gorsuch, R. L. y Lushene, R. E. (1970). Manual for the State-Trait Anxiety Inventory. Palo Alto, CA: Consulting Psychologists Press (versión española, TEA, 1994).

Taylor, G. y Bagby, R. (2000). An overview of the alexithymia construct. En R. Bar-On y J. Parker (Eds.), Handbook of emotional intelligence (pp. 4067). San Francisco: Jossey-Bass.

Taylor, G., Bagby, R. y Parker, J. (1997). Disorders of affect regulation: Alexithy mia in medical and psychiatric illness. Cambridge: Cambridge University Press.

Toro, J., Gómez-Peresmitré, G., Sentis, J., Vallés, A., Casulá, V., Castro, J. et al. (2006). Eating disorders and body image in Spanish and Mexican female adolescents. Social Psychiatry and Psychiatric Epidemiology, 41, 556565.

Veses, A. M., Martínez-Gómez, D., Gómez-Martínez, S., Zapatera, B., Veiga, O. L. y Marcos, A. (2010). The association between excessive body fat and Eating-disorder risk in adolescents: The AFINOS Study. Medicina Clínica, 136, 620-622.

Waller, G., Mountford, V., Lawson, R., Gray, E., Cordery, H. y Hinrichsen, H. (2010). Beating your eating disorder: A cognitive behavioral self-help guide for adult sufferers and their carers. New York: Cambridge University Press.

Wardle, J. y Cooke, L. (2005). The impact of obesity on psychological wellbeing in children. Best Practice \& Research: Clinical Endocrinoogy \& . Metabolism, 19, 421-440.

(Articulo recibido: 16-4-2012, revisado: 11-9-2012, aceptado: 26-9-2012) 
Apéndice 1. Se define como adolescentes con alto riesgo de desarrollar un TCA a aquellas que cumplen todos los criterios de, al menos, uno de los dos tipos siguientes:

\section{Posible riesgo de anorexia}

a. Cumplir, al menos, uno de los siguientes criterios:

a.1. Tener un índice de masa corporal de entre 17,51 y 18,49 .

a.2. Haber perdido $5 \mathrm{~kg}$. o más por dieta, con el objetivo de adelgazar, en el último año.

b. Cumplir, al menos, uno de los siguientes criterios:

b.1.Haber utilizado píldoras adelgazantes para controlar la figura o el peso (alguna vez en el último año).

b.2.Haber realizado ejercicio físico excesivo con el objetivo de controlar el peso, cambiar la figura o reducir la cantidad de grasa (durante las últimas 4 semanas). Se definió ejercicio físico excesivo como la realización de ejercicio físico durante más de una hora al día o más de 7 horas a la semana pero no todos los días.

b.3.Haber obtenido una puntuación $\geq 12$ en la Escala de Obsesión por la Delgadez del EDI-2.

b.4.Haber obtenido una puntuación $\geq 16$ en la Escala de Insatisfacción Corporal del EDI-2.

\section{Posible riesgo de purga/atracón}

a. Cumplir, al menos, uno de los siguientes criterios:

a.1. Haber realizado vómitos autoinducidos para controlar la figura o el peso (alguna vez en el último año).

a.2. Haber tomado laxantes para controlar la figura o el peso (alguna vez en el último año).

a.3. Haber utilizado diuréticos para controlar la figura o el peso (alguna vez en el último año).

a.4. Haber obtenido una puntuación $\geq 4$ en la Escala de Bulimia del EDI-2.

b. Cumplir, al menos, uno de los siguientes criterios:

b.1.Haber obtenido una puntuación $\geq 12$ en la Escala de Obsesión por la delgadez del EDI-2.

b.2.Haber obtenido una puntuación $\geq 16$ en la Escala de Insatisfacción corporal del EDI-2. 